\title{
Moral licensing: a culture-moderated meta-analysis
}

\author{
Philipp Simbrunner $^{1}$ - Bodo B. Schlegelmilch ${ }^{2}$
}

Received: 19 December 2016 / Accepted: 8 August 2017 / Published online: 16 August 2017 C The Author(s) 2017. This article is an open access publication

\begin{abstract}
Moral licensing is a cognitive bias, which enables individuals to behave immorally without threatening their self-image of being a moral person. We investigate this phenomenon in a cross-cultural marketing context. More specifically, this paper addresses the questions (i) how big moral licensing effects typically are and (ii) which factors systematically influence the size of this effect. We approach these questions by conducting a meta-analysis and a meta-regression. Based on a random effects model, the point estimate for the generalized effect size Cohen's $d$ is 0.319 ( $\mathrm{SE}=0.046 ; \mathrm{N}=106$ ). Results of a meta-regression advance theory, by showing for the first time that both cultural background and type of comparison explain a substantial amount of the total variation of the effect size of moral licensing. Marketing practitioners wishing to capitalize on moral licensing effects should therefore consider cross-cultural difference, since marketing measures building on this effect may lead to different revenues in different countries.
\end{abstract}

Keywords Moral licensing · Meta-analysis · Cross-cultural research · Behavioral economics $\cdot$ Cognitive bias

$凶 \quad$ Philipp Simbrunner

philipp.simbrunner@wu.ac.at

https://www.wu.ac.at/imm

Bodo B. Schlegelmilch

bodo.schlegelmilch@wu.ac.at

https://www.wu.ac.at/imm

1 Department of Marketing, Institute for International Marketing Management, Vienna University of Economics and Business, Welthandelsplatz 1, 1020 Vienna, Austria

2 Department of Marketing, Institute for International Marketing Management, Vienna University of Economics and Business \& Lingnan University College, Sun Yat-sen University,

Welthandelsplatz 1, 1020 Vienna, Austria 
JEL Classification M30 $\cdot$ M31 $\cdot$ M39

\section{Introduction}

Moral licensing is a non-conscious effect that provides a moral boost in the selfconcept, which increases the preference for a relative immoral action by dampening the negative self-attributions associated with such behavior (Khan and Dhar 2006). Applied to a marketing context, moral licensing, for example, explains why the purchase of a green product (a positive moral act) is likely to increase the probability of subsequently purchasing a self-indulgent luxury good (a negative moral act). While we do not consider luxury products as morally questionable per se, it has been shown that consumers often experience guilt after purchasing an expensive luxury item (Dahl et al. 2003). In a similar vein, it has been demonstrated that consumers repeatedly feel bad after selecting an indulgent good over a necessity (Kivetz and Simonson 2002).

Moral licensing affects consumer decisions, whenever one decision incorporates a positive moral dimension while a subsequent decision represents a comparatively negative moral dimension. Hence, the sequence in which such pairs of decisions are made influences the outcome of the latter. Especially in an online environment, retailers can easily control at which stage in the customer journey different product categories are presented to consumers. Based on moral licensing theory, companies should strive to present products with a positive moral dimension, like fair-traded or eco-friendly products, before enabling the purchase of self-indulgent items. Both consumers' willingness to pay and purchase likelihood for a luxury product should increase after having had the possibility to demonstrate social and/or ecological consciousness by purchasing a 'morally positive' product. Similarly, a moral licensing effect comes into play in the area of Corporate Social Responsibility (CSR). The engagement of companies in both cause-related marketing and philanthropy-oriented activities (Varadarajan and Menon 1988) is gaining importance and consumers' image of companies is shaped, at least partly, by CSR activities (Szőcs et al. 2016). One specific manifestation of CSR is the solicitation of donations, often in collaboration with a non-profit organization. For example, some airlines offer their customers the possibility to donate to an NGO during the ticket booking process in their online system. Links to donation options can be presented either before (i.e. on the start page) or after the actual purchase and payment for a company's products (i.e. after the purchase has been confirmed by the customer in the web shop). Moral licensing comes into effect through the contrast between the self-indulgent and the morally positive decision situation.

Moral licensing is a well-established cognitive bias and has been demonstrated in a variety of different contexts. The vast majority of existing research has focused on investigating whether the moral licensing effect occurs in differing scenarios. However, little knowledge exists on which factors drive the size of moral licensing effects. A recent attempt employed a meta-analytic approach (Blanken et al. 2015) to investigate influential factors but failed to identify any external moderators. Consequently, we aim to fill this research gap by focusing on hitherto neglected but potentially important moderators of moral licensing. Most importantly, we introduce, for the first time, cultural background as a pivotal factor for explaining variation in the strength of moral licens- 
ing. The potential impact of culture on the strength of moral licensing appears particularly relevant in that business is routinely crossing borders and cannot assume that consumers from different cultures are always following the same behavioral patterns.

We choose meta-analysis and meta-regression as a methodological approach due to several reasons. First, this approach allows us to draw our conclusions from a sample size that would be unmatched with techniques relying on primary data collection. Second, attempting to summarize systematically the entire literature body on the phenomenon, we are able to offer both a valuable overview on the topic and some well-grounded recommendations for future research directions. Third, we identified a need for the replication of an existing meta-analysis on moral licensing due to methodological issues encountered in that study.

Taken collectively, this paper aims to contribute to the further understanding of dynamics in human decision making by identifying factors, which systematically influence the size of moral licensing effects. In the following section, we further outline the phenomenon of moral licensing and introduce the potentially influential moderating factors we identified. Next, we offer a detailed description of our methodological approach and present the results of our analyses. Subsequently, we summarize the most important findings, discuss our insights in light of existing literature and provide both theoretical and managerial implications of our work. We conclude our paper by outlining detailed recommendations for future research directions.

\section{Theoretical background}

\subsection{Moral licensing effect, related cognitive biases and explanatory framework}

Moral licensing has been observed in a number of different settings. Monin and Miller (2001) were the first to systematically investigate and describe this phenomenon. They showed that individuals who signaled their egalitarianism by disagreeing with sexist statements were subsequently more likely to recommend a man for a traditionally male job. They argued that disagreeing with sexist statements helped allay individuals' concerns that their subsequent actions might be interpreted as morally questionable, thereby licensing their discriminatory behavior. Ever since, the existence of moral licensing effects has been demonstrated in numerous experiments that usually consist of two tasks: First, participants are given an opportunity to gain a moral license by displaying moral, pro-social behavior. In a second task, they have the possibility to demonstrate moral behavior as well. Having earned a moral license in the first decision situation, participants tend to show behavior that is more immoral in the second situation. Thus, in many cases, choices boosting one's self-concept subsequently appear to give a license for comparatively more self-indulgent or rather immoral choices. This pattern has been shown in a variety of differing domains. For example, Sachdeva et al. (2009) found that individuals who wrote self-reflective stories using a list of positive words like caring and generous would donate less money to charity and engage in fewer environmentally friendly actions than individuals who wrote self-critical stories using negative words like greedy and disloyal. Similarly, Khan and Dhar (2006) found that people who imagined performing a pro-social task like donating money or 
volunteering time were subsequently more likely to choose a luxury over a utilitarian product. In a similar vein, Mazar and Zhong (2010) investigated this phenomenon in the context of environmental friendliness. Two groups of goods were offered to participants: environmentally friendly goods and non-green goods. Individuals who chose from the green product portfolio were more prone to lying or stealing money in a subsequent task than individuals who chose among non-green products. Thus, research on moral licensing suggests that moral actions can lead individuals to relax their moral standards and engage in less moral behavior.

Two related yet different cognitive biases are known, namely moral cleansing and prospective moral licensing. First, moral cleansing occurs when an individual's overall moral self-perception has been endangered by displaying or recalling immoral behavior. Consequently, the likelihood of showing moral behavior subsequently increases in order to reestablish a moral image of one's self (Jordan et al. 2011). Second, prospective moral licensing enables individuals to display immoral behavior in the present, if they know that they will have the opportunity to demonstrate moral behavior in the future. Cascio and Plant (2015), for example, showed that expressing one's plans to donate blood in the future increases the likelihood of stating racist attitudes in the present. Despite being related theoretically, both aforementioned phenomena are beyond the scope of this research. This paper purely focuses on moral licensing.

The existence of moral licensing (as well as moral cleansing and prospective moral licensing) seems to contradict several well-established psychological theories, which stress the importance of consistency in an individual's behavior and thus an individual's tendency to maintain consistency in one's moral identity (Colby and Damon 1993). According to self-perception theory (Bem 1972), for example, individuals form attitudes about themselves by deducting these attitudes from their own prior behavior and this perception of one's self ultimately influences subsequent behavior. Hence, performing a pro-social task should foster one's self-perception as a moral person and should consequently encourage consistent behavior. Similarly, other wellestablished psychological theories such as cognitive dissonance theory (Festinger 1957) or balance theory (Heider 1946) stress consistency in one's own judgments and decisions as one key element of human behavior. However, one potential explanation for moral licensing considers this phenomenon as one smaller element of a larger self-regulation framework (Sachdeva et al. 2009). Individuals establish a moral self-image throughout their lifespan. Regardless of whether one perceives oneself as a highly moral or less moral person, this established self-image provides a moral baseline. Sachdeva et al. (2009) suggest that by displaying moral behavior, an individual reinforces her/his moral self-image. Hence, the perceived degree of "being moral" exceeds the individual-specific baseline. Therefore, showing comparatively immoral behavior subsequently does not put the overall moral self-image under threat, and hence leads to moral licensing.

\subsection{Prior attempts to identify moderators}

While the existence of the moral licensing effect is well known, the extant literature offers only few insights on what may drive the size of moral licensing effects. In a 
recently published meta-analysis, Blanken et al. (2015) showed that the generalized size of the moral licensing effect is Cohen's $d=0.31$, where over $50 \%$ of the variation in effect sizes between single study outcomes is due to a systematic between-study variance and not due to sampling errors. This relatively high amount of unexplained variation in size calls for the identification of influential factors. Below, we provide an overview over moderators that have been under investigation by prior research.

\subsection{Type of induction}

A moral license can be induced by either displaying/recalling a moral action or by displaying/recalling a moral trait. Theoretically, moral actions can be seen as one step towards the overall goal of being a moral person, whereas moral traits can be seen as one integral part of one's moral self-identity (Conway and Peetz 2012). By recalling or displaying moral actions, the goal of being a moral person is further accomplished and therefore a subsequent immoral behavior is more likely to occur. By recalling or stating moral traits, acting consistently with one's moral self-image becomes more important and hence immoral behavior in a subsequent choice is less likely to occur. An example for inducing a moral license via a moral action is to ask participants about donating money to charity (e.g. Khan and Dhar 2006), whereas an example for a license induction via moral traits is to ask participants to write self-reflective stories about themselves using positive words such as generous or caring (e.g. Sachdeva et al. 2009).

\subsection{Type of behavior}

Measured behavior in the second task can be either actual or hypothetical behavior. For example, in a hypothetical scenario (Effron et al. 2012) asked participants to state their willingness to hire a white person over a black person. On the contrary, Cornelissen et al. (2013a) measured the amount of money offered by participants in an actual dictator game. Previous research demonstrated that individuals want to appear moral whilst trying to avoid the costs of being moral (Batson and Thompson 2001; Dana et al. 2007). Therefore, hypothetical versus actual decisions should result in differently sized moral licensing effects.

\subsection{Domain in which behavior is observed}

Both decision situations can be either within the same or within different domains. Monin and Miller (2001), for example, offered participants the possibility to disagree with obviously sexist statements and subsequently asked them to indicate whether they would rather hire a man or a woman for a "typical" male job. In Khan and Dhar's (2006) experiment, participants were asked to imagine donating money or time to charity and subsequently had the opportunity to choose between a utilitarian and a hedonic good. Mental accounting theory (Thaler 1985) suggests that individuals are more likely to demonstrate immoral behavior in the same domain. 


\subsection{Control condition}

The moral licensing condition in which participants are asked to perform or recall a good deed can be either compared to a neutral condition, in which participants perform or recall neutral behavior, or an immoral condition, in which participants perform or recall a bad deed. This is important from a theoretical perspective, as the opposite pattern of moral cleansing exists as well (e.g. Zhong and Liljenquist 2006). One would therefore expect a bigger moral licensing effect size when comparing the experimental group to an immoral condition as opposed to a neutral condition.

\subsection{Publication status}

Given the nature of this moderator, it can be used solely in a meta-analytic approach and is not suitable for primary data. Publication status denotes whether the data included in the analysis either was published in a journal article or was unpublished data obtained through personal contact with researchers in this field (Cooper 2010).

In an attempt to identify moderators, which systematically influence the size of the moral licensing effect, Blanken et al. (2015) investigated the five moderators discussed in this section. However, they found that the publication status was the only statistically significant predictor in a meta-regression analysis. None of the other aspects was able to explain systematic variation in effect sizes. For a substantial part of effect size variance, the source of variation remains unclear. Consequently, our paper aims at contributing to the understanding of what drives the strength of moral licensing. More specifically, we suggest four potentially influential moderating aspects: cultural background, type of decision, type of comparison, and publication status. We explore the role of these moderators by conducting a meta-analysis and meta-regressions.

\subsection{Expected contribution}

In a previous study, Blanken et al. (2015) focused on the identification of the generalized effect size of moral licensing, but were unable to identify any study-specific or sample-specific factors, which moderate the strength of moral licensing. Our paper builds on these results and now explicitly emphasizes the identification of influential moderators. Specifically, we consider cross-cultural differences as the pivotal aspect in our analysis. To the best of our knowledge, we are the first to investigate the moral licensing effect in a cross-cultural context. In addition, we make a conceptual contribution by focusing purely on moral licensing, whereas Blanken et al. (2015) also included data from primary studies investigating prospective moral licensing. Although both concepts are related, we propose to make a distinction between them, because by definition they describe distinct phenomena. Hence, our meta-analysis on moral licensing purely relies on data obtained through primary studies explicitly designed as moral licensing experiments. By using meta-analysis and meta regression, we also aim to make a methodological contribution, in that this approach enables us to draw inferences from a sample size that far surpasses any individual primary data collection effort. Furthermore, meta-analyses typically include an attempt to summarize sys- 
tematically the entire body of literature on a given phenomenon. This enables us to offer a valuable overview on moral licensing at large and to present well-grounded recommendations for future research directions.

\subsection{Conceptualization of moderators}

In the following section, we outline the potential relevance of these moderators and provide a detailed discussion of each moderator's hypothesized impact on moral licensing effect sizes. Given the nature of meta-analyses, we are constrained in the selection of moderators by the information reported in primary studies. The moderators we chose were both reported consistently across identified primary studies and show a substantial amount of variation between studies. Views on morality differ across cultures (Pew Research Center 2014), hence we assume an impact of culture on moral licensing. Building on self-gratification theory (Baumann et al. 1981), we suggest that moral licenses have a bigger impact on self-rewarding than on society-related decisions. Due to the phenomenon of moral cleansing (Conway and Peetz 2012), contrasting a moral licensing group with a neutral control group should result in smaller effect sizes than the comparison with a group whose participants performed an immoral task. Finally and in line with prior research (Blanken et al. 2015) we expect that published studies show a bigger effect size than unpublished studies.

\subsection{Cultural background}

We put a pivotal emphasize on the investigation of cross-cultural differences. According to the explanatory framework of self-regulation (Sachdeva et al. 2009), the strength of the moral licensing effect is rooted in an individual's self-concept with regard to morality. This self-concept, in turn, is based on the understanding of morality derived from an individual's cultural background (Ward 2014). Moral standards are acquired through socialization processes and hence, are highly dependent on the cultural environment an individual grows up in and interacts with throughout her/his lifespan. Therefore, we consider cultural background as an important influencer of moral licensing. Moreover, behavioral economist recently called for research on cultural differences in cognitive biases (e.g. Samson 2016).

Moral licensing experiments have been conducted on a variety of diverse issues, with diverse moral dimensions ranging from racism (e.g. Effron et al. 2009) and sexism (e.g. Monin and Miller 2001) over selfishness (e.g. Sachdeva et al. 2009) to environmental friendliness (e.g. Mazar and Zhong 2010). Focusing on the cultural background, members of culturally distinct world regions differ in their views on issues related to questions of morale (Pew Research Center 2014). More specifically, very few Europeans rate the role of religiosity in their life as very important as compared to the ascribed role of religion in other world regions such as North America or South-East Asia. For example, only $13.1 \%$ of Germans state that religion plays an important role in their life, whereas $40.4 \%$ of US Americans and $56.5 \%$ of Thais do so (World Values Survey 2014). Similarly, across the globe, the perceived importance of unselfishness as an important child quality differs. Whereas, for example, $44.3 \%$ of 
Thai and $32.7 \%$ of US American respondents agree that unselfishness is an important trait to teach children, only $5.9 \%$ of Germans share this view (Ward 2014). Consequently, we hypothesize that moral licensing effects differ in size between culturally distinct world regions. More precisely, we assume that the effect is larger in samples consisting of participants from countries, where questions of morale are perceived as more central to an individual's identity. Thus, we expect moral licensing effects to be bigger, for example, in studies from the USA and smaller in studies from Germany.

\subsection{Type of decision}

In terms of the type of decision in moral licensing effect experiments, the second task, which is used to measure the strength of the moral license gained in the first task, can be either rather related to one's self or rather related to society at large. An example for a rather self-related decision task is having the possibility to cheat in an exam, where having gained a moral license leads to an increased likelihood of cheating (e.g. Zhong et al. 2010). An example for a rather society-related decision task is having the possibility to donate money to charity, where experiencing moral licensing results in a decreased average amount of money donated (e.g. Young et al. 2012). Having performed a moral action in the first task, i.e. having gained a moral license, leads to a boost in one's self-concept (Khan and Dhar (2006). This affirmative boost puts individuals in a positive mood, which in turn increases an individual's tendency for self-gratification seeking (Baumann et al. 1981). Arguably, pro-social behavior, which benefits society, can be seen as self-rewarding as well. However, we consider tasks comprising also a pro-social dimension as less self-rewarding in the sense of self-indulgent as compared to behavior that purely benefits one's self. Hence, we assume a difference in effect sizes between self-related and society-related decisions. Moral licensing should foster non-moral behavior more strongly, if the outcome of the decision is of benefit to an individual as compared to a benefit to society.

\subsection{Type of comparison}

The behavior of the moral license group is compared either with a neutral control group or with a second experimental group, which is asked to perform an immoral task in the first place. This is important from a theoretical point of view, as the opposite pattern can exist as well: Displaying or recalling immoral behavior can increase the likelihood of displaying moral behavior subsequently. This phenomenon is known as moral cleansing (Conway and Peetz 2012). Hence, we assume a difference in moral licensing effect sizes between these two experimental designs. The effect should be bigger when an immoral control group is used and smaller when a neutral control condition is used. Blanken et al. (2015) investigated the influence of the comparison between immoral and neutral conditions and could not find any significant differences. This result is surprising and counter-intuitive from a methodological perspective, since we would expect that differently designed control groups lead to different effect sizes. We will have a fresh look at this issue, as our data set includes a more comprehensive and fine-grained set of studies. 


\subsection{Publication status}

Finally, Blanken et al. (2015) identified a study's publication status as potential influential factor of effect's size. Published data obtained from journal articles tended to show bigger effect sizes than unpublished data obtained through personal contact. Unsurprisingly, unpublished data tends to show smaller effect sizes or no statistically significant effect at all, which at least partly is a reason for this data not being published (e.g. Cooper 2010). We include this aspect in our analysis for two reasons. First, in order to validate findings from previous research and second, to use this information as a control variable.

\section{Method}

We conduct a meta-analysis in order to identify the overall, generalized effect size for moral licensing. As effect size measure, we use Cohen's $d$ values. Additionally, all moderators described above are used in a meta-regression model. All calculations are performed with the specialized software Comprehensive Meta-Analysis (2014).

\subsection{Data appraisal}

We identified relevant studies through online searches on ProQuest, EBSCO, and Web of Science. The following keywords were used: moral licensing, self-licensing, moral credentials, moral credential, moral self-licensing, credentialing, moral licenses and moral license. When deciding whether to include a particular study, we relied on the definition of moral licensing presented above. Purely theoretical papers were not included. Furthermore, we excluded studies focusing on moral cleansing, i.e. showing immoral behavior in the first task with the known opportunity to clean this immoral behavior in a subsequent task by displaying moral behavior (Jordan et al. 2011). In addition, we excluded studies focusing on prospective moral licensing, i.e. planning on performing moral behavior in the future, which affects the morality of displayed behavior in the first task (Cascio and Plant 2015; Merritt et al. 2012). Overall, we identified 23 published journal articles, the oldest being from 2001. In these articles, 61 single study outcomes are given and used for our research. Moreover, we included 34 single study outcomes reported by Blanken et al. (2015) as well as 11 additional, unpublished single study outcomes. Hence, we were able to analyze 106 effect sizes in total.

\subsection{Coding process}

In the included studies the dependent variables measured are either a decrease in moral behavior, such as donating less money to charity (e.g. Sachdeva et al. 2009), or an increase in immoral behavior, such as cheating or stealing (Mazar and Zhong 2010). For our analyses, all effect sizes were recoded accordingly so that a positive effect size indicates a decrease in moral behavior after performing a moral task in the first place. 
For calculating the effect size, we use means, standard deviations and sample sizes of the experimental and the control condition, respectively. When information on means and standard deviations are not reported along with primary data, we use $t$-values for the calculations. Calculations were performed with Comprehensive Meta-Analysis (2014), following the equations for computing effect sizes described in e.g. Lipsey and Wilson (2001) or Cooper (2010). Some studies report a comparison of the moral license condition with both a neutral and an immoral control group. In these cases, we always use the comparison with the neutral control group for calculating the effect size. Table 1 depicts all coded effect sizes and moderators for each study included in our analyses.

The three moderators labeled type of comparison, type of decision and publication status are dummy-coded. For capturing the information of cultural background, we first coded the country in which the respective study was conducted. In a second step, we collapsed the countries into bigger regions, namely Western Europe, North America and South-East Asia. We could not identify studies conducted in other world regions besides these three. We are fully aware of the fact that using countries as source of information for cultural background does not necessarily capture information about the actual cultural background of individuals who participated in primary studies. While recognizing this constraint, we also observe that employing meta-analytical approaches with countries or groups of countries as moderators is a well-established research strategy in cross-cultural psychology (Bond and Smith 1996; Khaleque and Rohner 2002; Mezulis et al. 2004; Twenge and Campbell 2002; Watkins 2001). Since primary data collection in cross-cultural research is both time consuming and cost intensive, we consider employing a meta-analytic approach as a resource-efficient option for identifying (first) evidence of cultural differences in a phenomenon of interest.

\subsection{Analyses}

We perform a meta-analysis with the computed effect sizes applying a random effects model. The potential influence of coded moderators is analyzed in two ways. First, we use each dummy-coded moderator separately in a subgroup analysis. In a next step, we run meta-regression models using all possible combinations of moderators. In addition to using the publication status as a moderator, we also address the issue of publication bias (e.g. Borenstein et al. 2013) by means of a fail-safe-N calculation.

\section{Results}

\subsection{Meta-analysis}

To identify the overall generalized effect size of moral licensing, we run a random effects model. Generalized effect sizes in a random effects model assume that in addition to sampling error, differences between single study outcomes are due to the systematic influence of at least one additional moderator. In contrast, in a fixed effects model the computed generalized effect size is based on the assumption that differences 
Table 1 Details of all studies included in the meta-analysis

\begin{tabular}{|c|c|c|c|c|c|c|c|c|c|}
\hline Authors & Study & $\mathrm{N}$ & d & SE & A & $B$ & $\mathrm{C}$ & $\mathrm{D}$ & $\mathrm{E}$ \\
\hline Blanken et al. (2012) & 1 & 86 & -0.08 & 0.22 & 1 & 0 & 0 & NL & EUR \\
\hline Blanken et al. (2012) & 2 & 86 & 0.07 & 0.22 & 1 & 0 & 0 & NL & EUR \\
\hline Blanken et al. (2012) & 3 & 38 & 0.05 & 0.32 & 1 & 1 & 0 & NL & EUR \\
\hline Blanken et al. (2012) & 4 & 49 & 0.49 & 0.29 & 1 & 1 & 0 & NL & EUR \\
\hline Blanken et al. (2012) & 5 & 47 & 0.03 & 0.29 & 1 & 1 & 0 & NL & EUR \\
\hline Blanken et al. (2012) & 6 & 54 & 0.30 & 0.27 & 1 & 0 & 0 & NL & EUR \\
\hline Blanken et al. (2012) & 7 & 65 & 0.54 & 0.25 & 1 & 0 & 0 & NL & EUR \\
\hline Blanken et al. (2012) & 8 & 57 & -0.11 & 0.27 & 1 & 1 & 0 & NL & EUR \\
\hline Blanken et al. (2012) & 9 & 53 & 0.12 & 0.27 & 1 & 1 & 0 & NL & EUR \\
\hline Blanken et al. (2012) & 10 & 50 & 0.61 & 0.29 & 1 & 0 & 0 & NL & EUR \\
\hline Blanken et al. (2012) & 11 & 49 & 0.48 & 0.29 & 1 & 0 & 0 & NL & EUR \\
\hline Blanken et al. (2012) & 12 & 48 & 0.63 & 0.30 & 1 & 0 & 0 & NL & EUR \\
\hline Blanken et al. (2012) & 13 & 51 & 0.22 & 0.28 & 1 & 0 & 0 & $\mathrm{NL}$ & EUR \\
\hline Blanken et al. (2012) & 14 & 94 & 0.86 & 0.22 & 1 & 1 & 0 & $\mathrm{NL}$ & EUR \\
\hline Blanken et al. (2012) & 15 & 133 & 0.24 & 0.17 & 1 & 1 & 0 & NL & EUR \\
\hline Blanken et al. (2012) & 16 & 65 & -0.19 & 0.25 & 1 & 0 & 0 & NL & EUR \\
\hline Blanken et al. (2012) & 17 & 61 & 0.04 & 0.26 & 1 & 0 & 0 & $\mathrm{NL}$ & EUR \\
\hline Blanken et al. (2012) & 18 & 61 & -0.34 & 0.26 & 1 & 1 & 0 & NL & EUR \\
\hline Blanken et al. (2012) & 19 & 83 & -0.25 & 0.22 & 1 & 1 & 0 & NL & EUR \\
\hline Blanken et al. (2012) & 20 & 57 & -0.67 & 0.27 & 1 & 1 & 0 & NL & EUR \\
\hline Blanken et al. (2014) & 1 & 64 & 0.08 & 0.25 & 1 & 1 & 1 & NL & EUR \\
\hline Blanken et al. (2014) & 2 & 91 & 0.27 & 0.21 & 1 & 1 & 1 & NL & EUR \\
\hline Blanken et al. (2014) & 3 & 614 & -0.03 & 0.08 & 1 & 0 & 1 & US & NOA \\
\hline Blanken et al. (2014) & 4 & 614 & -0.07 & 0.08 & 1 & 1 & 1 & US & NOA \\
\hline Bradley-Geist et al. (2010) & 1 & 38 & -0.17 & 0.33 & 1 & 1 & 1 & US & NOA \\
\hline Bradley-Geist et al. (2010) & 2 & 35 & 0.66 & 0.35 & 1 & 1 & 1 & US & NOA \\
\hline Bradley-Geist et al. (2010) & 3 & 44 & 0.17 & 0.30 & 1 & 1 & 1 & US & NOA \\
\hline Bradley-Geist et al. (2010) & 4 & 42 & 0.47 & 0.31 & 1 & 1 & 1 & US & NOA \\
\hline Bradley-Geist et al. (2010) & 5 & 56 & -0.70 & 0.28 & 0 & 1 & 1 & US & NOA \\
\hline Brown et al. (2011) & 1 & 97 & 0.41 & 0.21 & 1 & 0 & 1 & US & NOA \\
\hline Cain et al. (2005) & 1 & 43 & 0.58 & 0.31 & 1 & 0 & 1 & US & NOA \\
\hline Cain et al. (2011) & 1 & 348 & -0.15 & 0.11 & 1 & 0 & 1 & US & NOA \\
\hline Cain et al. (2011) & 2 & 49 & 0.04 & 0.29 & 1 & 0 & 1 & US & NOA \\
\hline Cain et al. (2011) & 3 & 77 & 0.40 & 0.23 & 1 & 0 & 1 & US & NOA \\
\hline Choi et al. (2014) & 1 & 116 & 0.44 & 0.19 & 1 & 1 & 1 & US & NOA \\
\hline Choi et al. (2014) & 2 & 60 & 0.54 & 0.26 & 1 & 1 & 1 & US & NOA \\
\hline Clot et al. (2013a) & 1 & 367 & 0.23 & 0.10 & 1 & 1 & 0 & FR & EUR \\
\hline Clot et al. (2013b) & 1 & 192 & 0.30 & 0.15 & 1 & 1 & 1 & FR & EUR \\
\hline Clot et al. (2014) & 1 & 100 & 0.35 & 0.20 & 1 & 0 & 1 & FR & EUR \\
\hline Conway and Peetz (2012) & 1 & 51 & 0.33 & 0.28 & 0 & 1 & 1 & US & NOA \\
\hline
\end{tabular}


Table 1 continued

\begin{tabular}{|c|c|c|c|c|c|c|c|c|c|}
\hline Authors & Study & $\mathrm{N}$ & d & SE & A & $B$ & $\mathrm{C}$ & $\mathrm{D}$ & $\mathrm{E}$ \\
\hline Conway and Peetz (2012) & 2 & 39 & 0.58 & 0.33 & 0 & 1 & 1 & US & NOA \\
\hline Conway and Peetz (2012) & 3 & 65 & 0.00 & 0.25 & 0 & 1 & 1 & US & NOA \\
\hline Conway and Peetz (2012) & 4 & 65 & 0.79 & 0.26 & 0 & 1 & 1 & US & NOA \\
\hline Cornelissen et al. (2013a) & 1 & 48 & 0.59 & 0.29 & 0 & 0 & 1 & $\mathrm{DE}$ & EUR \\
\hline Cornelissen et al. (2013a) & 2 & 40 & 0.84 & 0.33 & 0 & 0 & 1 & $\mathrm{DE}$ & EUR \\
\hline Cornelissen et al. (2013a) & 3 & 50 & 0.53 & 0.29 & 0 & 0 & 1 & $\mathrm{DE}$ & EUR \\
\hline Cornelissen et al. (2013b) & 1 & 70 & 0.57 & 0.24 & 0 & 1 & 0 & $\mathrm{DE}$ & EUR \\
\hline Cornelissen et al. (2013b) & 2 & 92 & 0.36 & 0.21 & 0 & 1 & 0 & $\mathrm{DE}$ & EUR \\
\hline Effron et al. (2009) & 1 & 84 & 0.44 & 0.22 & 1 & 1 & 1 & US & $\mathrm{NOA}$ \\
\hline Effron et al. (2009) & 2 & 40 & 0.35 & 0.32 & 1 & 1 & 1 & US & NOA \\
\hline Effron et al. (2009) & 3 & 50 & 0.16 & 0.28 & 1 & 1 & 1 & US & $\mathrm{NOA}$ \\
\hline Effron (2014) & 1 & 107 & 0.44 & 0.20 & 1 & 1 & 1 & US & NOA \\
\hline Effron (2014) & 2 & 106 & 0.47 & 0.20 & 1 & 0 & 1 & US & NOA \\
\hline Effron et al. (2012) & 1 & 157 & 0.28 & 0.16 & 1 & 1 & 1 & US & NOA \\
\hline Effron et al. (2012) & 2 & 157 & 0.20 & 0.16 & 1 & 1 & 1 & US & NOA \\
\hline Jordan et al. (2011) & 1 & 51 & 1.15 & 0.30 & 0 & 1 & 1 & US & NOA \\
\hline Jordan et al. (2011) & 2 & 84 & 1.00 & 0.23 & 0 & 0 & 1 & US & NOA \\
\hline Jordan et al. (2011) & 3 & 84 & 1.01 & 0.23 & 0 & 0 & 1 & US & $\mathrm{NOA}$ \\
\hline Khan and Dhar (2006) & 1 & 108 & 0.62 & 0.20 & 1 & 0 & 1 & US & NOA \\
\hline Khan and Dhar (2006) & 2 & 94 & 0.60 & 0.21 & 1 & 0 & 1 & US & NOA \\
\hline Khan and Dhar (2006) & 3 & 80 & 0.56 & 0.23 & 1 & 0 & 1 & US & NOA \\
\hline Khan and Dhar (2006) & 4 & 80 & 0.39 & 0.23 & 1 & 1 & 1 & US & NOA \\
\hline Khan and Dhar (2006) & 5 & 80 & 0.46 & 0.23 & 1 & 0 & 1 & US & NOA \\
\hline Khan and Dhar (2006) & 6 & 66 & 0.56 & 0.25 & 1 & 0 & 1 & US & NOA \\
\hline Kouchaki (2011) & 1 & 64 & 0.58 & 0.26 & 1 & 1 & 1 & US & NOA \\
\hline Kouchaki (2011) & 2 & 64 & 0.57 & 0.26 & 1 & 0 & 1 & US & NOA \\
\hline Kouchaki (2011) & 3 & 96 & 0.52 & 0.21 & 1 & 1 & 1 & US & NOA \\
\hline Kouchaki (2011) & 4 & 50 & 0.64 & 0.29 & 1 & 1 & 1 & US & NOA \\
\hline Leonard (2012) & 1 & 32 & -0.29 & 0.36 & 1 & 0 & 0 & NA & NA \\
\hline Leonard (2012) & 2 & 92 & -0.04 & 0.21 & 0 & 0 & 0 & NA & NA \\
\hline Leonard (2012) & 3 & 43 & -0.02 & 0.31 & 0 & 0 & 0 & NA & NA \\
\hline Leonard (2012) & 4 & 64 & 0.01 & 0.25 & 0 & 1 & 0 & NA & NA \\
\hline Mann and Kawakami (2012) & 1 & 44 & 0.73 & 0.31 & 0 & 0 & 1 & US & NOA \\
\hline Mann and Kawakami (2012) & 2 & 94 & 0.55 & 0.21 & 0 & 1 & 1 & US & NOA \\
\hline Mann and Kawakami (2012) & 3 & 30 & 0.86 & 0.38 & 0 & 0 & 1 & US & NOA \\
\hline Mazar and Zhong (2010) & 1 & 76 & 0.29 & 0.23 & 0 & 0 & 1 & $\mathrm{CA}$ & NOA \\
\hline Mazar and Zhong (2010) & 2 & 80 & 3,19 & 0.34 & 0 & 0 & 1 & $\mathrm{CA}$ & NOA \\
\hline Mazar and Zhong (2010) & 3 & 80 & 3,55 & 0.36 & 0 & 0 & 1 & $\mathrm{CA}$ & NOA \\
\hline Meijers et al. (2014) & 1 & 40 & -0.04 & 0.32 & 1 & 1 & 0 & NL & EUR \\
\hline Meijers et al. (2014) & 2 & 40 & 0.83 & 0.33 & 1 & 1 & 0 & NL & EUR \\
\hline
\end{tabular}


Table 1 continued

\begin{tabular}{|c|c|c|c|c|c|c|c|c|c|}
\hline Authors & Study & $\mathrm{N}$ & $\mathrm{d}$ & SE & A & $B$ & $\mathrm{C}$ & $\mathrm{D}$ & $\mathrm{E}$ \\
\hline Meijers et al. (2014) & 3 & 88 & 0.60 & 0.22 & 1 & 1 & 0 & $\mathrm{NL}$ & EUR \\
\hline Meijers et al. (2014) & 4 & 88 & -0.24 & 0.21 & 1 & 1 & 0 & $\mathrm{NL}$ & EUR \\
\hline Monin and Miller (2001) & 1 & 140 & 0.26 & 0.17 & 1 & 1 & 1 & US & NOA \\
\hline Monin and Miller (2001) & 2 & 110 & 0.71 & 0.20 & 1 & 1 & 1 & US & NOA \\
\hline Monin and Miller (2001) & 3 & 20 & 0.91 & 0.47 & 1 & 1 & 1 & US & NOA \\
\hline Sachdeva et al. (2009) & 1 & 29 & 0.60 & 0.38 & 1 & 0 & 1 & US & NOA \\
\hline Sachdeva et al. (2009) & 2 & 18 & 1.10 & 0.51 & 0 & 0 & 1 & US & NOA \\
\hline Sachdeva et al. (2009) & 3 & 31 & 0.57 & 0.37 & 1 & 1 & 1 & US & NOA \\
\hline Schüler et al. (2012) & 1 & 62 & -0.42 & 0.26 & 1 & 0 & 0 & $\mathrm{DE}$ & EUR \\
\hline Spektor (2014) & 1 & 278 & 0.00 & 0.12 & 0 & 1 & 0 & $\mathrm{DE}$ & EUR \\
\hline Susewind and Hoelzl (2014) & 1 & 84 & 0.38 & 0.22 & 1 & 1 & 1 & $\mathrm{DE}$ & EUR \\
\hline Susewind and Hoelzl (2014) & 2 & 62 & 0.44 & 0.26 & 0 & 0 & 1 & DE & EUR \\
\hline Thomas and Showers (2012) & 1 & 76 & -0.30 & 0.23 & 0 & 0 & 0 & NA & NA \\
\hline Young et al. (2012) & 1 & 66 & -0.41 & 0.25 & 1 & 1 & 1 & US & NOA \\
\hline Zhong et al. (2010) & 1 & 68 & 0.29 & 0.24 & 1 & 0 & 1 & US & NOA \\
\hline (Simbrunner and Schlegelmilch 2016a) & 1 & 52 & 0.66 & 0.29 & 1 & 0 & 0 & AT & EUR \\
\hline (Simbrunner and Schlegelmilch 2016a) & 1 & 57 & -0.16 & 0.27 & 1 & 0 & 0 & AT & EUR \\
\hline (Simbrunner and Schlegelmilch 2016a) & 2 & 57 & 0.26 & 0.27 & 1 & 0 & 0 & AT & EUR \\
\hline (Simbrunner and Schlegelmilch 2016a) & 3 & 57 & 0.35 & 0.27 & 1 & 0 & 0 & AT & EUR \\
\hline (Simbrunner and Schlegelmilch 2016a) & 4 & 57 & 0.58 & 0.27 & 1 & 0 & 0 & AT & EUR \\
\hline (Simbrunner and Schlegelmilch 2016a) & 5 & 57 & 0.54 & 0.27 & 1 & 0 & 0 & AT & EUR \\
\hline (Simbrunner and Schlegelmilch 2016a) & 1 & 111 & -0.26 & 0.19 & 1 & 0 & 0 & $\mathrm{TH}$ & SEA \\
\hline (Simbrunner and Schlegelmilch 2016a) & 2 & 111 & -0.49 & 0.19 & 1 & 0 & 0 & $\mathrm{TH}$ & SEA \\
\hline (Simbrunner and Schlegelmilch 2016a) & 3 & 111 & -0.32 & 0.19 & 1 & 0 & 0 & $\mathrm{TH}$ & SEA \\
\hline (Simbrunner and Schlegelmilch 2016a) & 4 & 111 & -0.38 & 0.19 & 1 & 0 & 0 & $\mathrm{TH}$ & SEA \\
\hline (Simbrunner and Schlegelmilch 2016a) & 5 & 111 & -0.41 & 0.19 & 1 & 0 & 0 & $\mathrm{TH}$ & SEA \\
\hline
\end{tabular}

$N$ number of participants in this study, $d$ Cohen's d effect size, $S E$ standard error, $A$ type of comparison: immoral (0) versus neutral (1) control condition, $B$ type of decision: self-related ( 0$)$ versus society-related (1) decision, $C$ publication status: unpublished (0) versus published (1) data, $D$ country in which study was conducted: $A T$ Austria, $C A$ Canada, DE Germany, FR France, $N L$ Netherlands, $T H$ Thailand, USA USA, $N A$ no information, $E$ world region: EUR Western Europe, NOA North America, SEA South-East Asia

between single study outcomes are only due to sampling errors (e.g. Cooper 2010). Based on our theoretical knowledge, we choose a random effects model as the appropriate model for our analysis. Figure 1 depicts the forest plot of the meta-analysis. For the random effects model, the point estimate for the generalized effect size Cohen's $d$ is $0.319(S E=0.046 ; p<0.000 ; N=106)$ with lower and upper confidence interval limits of 0.229 and 0.408 , respectively. The point estimate is significantly different from zero. In order to address the robustness of the result, we perform a classical fail-safe-N (Rosenthal 1979) test. This calculation indicates the number of additional non-significant single studies needed in order to bring the computed generalized effect 
Bradley-Geist, J. C., King, E. B., Skorinko, J., Hebl, M. R., \& McKenna, C. (2010). E Blanken, I., Van de Ven, N., \& Zeelenberg, M. (2012). T

Simbrunner, P., \& Schlegelmilch, B. B. (2016), H

Schueler, S., Lehnhardt, N., \& Huber, M. (2012)
Young, L., Chakroff, A., \& Tom, J. (2012). A

Young, L., Chakroff, A., \& Tom, J. (2012). A
Simbrunner, P., \& Schlegelmilch, B. B. (2016), K

Blanken, I., Van de Ven, N., \& Zeelenberg. M (2012) R

Simbrunner, P., \& Schlegelmilch, B. B. (2016), I
Thomas, J. S., \& Showers, C. (2012). A

Leonard, B. (2012). A

Simbrunner, P., \& Schlegelmilch, B. B. (2016), G

Blanken, L., Van de Ven, N., \& Zeelenberg, M. (2012). S
Meijers, M. H. C., Noordewier, M. K., Verlegh, P. W. J., \& Smit, E. G. (2014). D Blanken, I. ., Van de Ven, N., \& Zeelenberg, M. (2012). P
Bradley-Geist, J. C., King, E. B., Skorinko, J., Hebl, M. R., \& McKenna, C. (2010). A

Simbrunner, P., \& Schlegelmilch, B. B. (2016), B

Cain, D. M., Loewenstein, G., \& Moore, D. A. (2011). A

Blanken, L., Van de Ven, N. \& Zeelenberg, M. (2012). H

Blanken, I., Van de Ven, N., \& Zeelenberg, M. (2012). A
Blanken, I., van de Ven, N., Zeelenberg, M., \& Meijers, M. H. (2014). D

Leonard, B. (2012). B

Meijers, M. H. C., Noordewier, M. K., Verlegh, P. W. J., \& Smit, E. G. (2014). A

Blanken, l., van de Ven, N., Zeelenberg, M., \& Meijers, M. H. (2014). C

Leonard, B. (2012). $C$

Conway, P., \& Peetz, J. (2012). C

Spektor, M. (2014). A

Blanken, I., Van de Ven, N., \& Zeelenberg, M. (2012). E
Blanken, I., Van de Ven, N., \& Zeelenberg, M. (2012). Q

Cain, D. M., Loewenstein, G., \& Moore, D. A. (2011). B

Blanken, I., Van de Ven, N., \& Zeelenberg, M. (2012). C
Blanken, I., Van de Ven, N., \& Zeelenberg, M. (2012). B

Blanken, I., van de Ven, N., Zeelenberg, M., \& Meijers, M. H. (2014). A

Blanken, I., van de Ven, N., Zeelenberg, M., \& Mejers, M.
Blanken, I., Van de Ven, N., \& Zeelenberg, M. (2012). I

Blanken, l., Van de Ven, N., \& Zee

Bradley-Geist, J. C.. King, E. B., Skorinko, J., Hebl, M. R., \& McKenna, C. (2010) C

Effron, D. A. Miller, D. T., \& Monin, B. (2012).

Blanken, I., Van de Ven, N., \& Zeelenberg, M. (2012). M

Clot, S., Grolleau, G., \& Ibanez, L. (2013). A

Blanken, I., Van de Ven, N., \& Zeelenberg, M. (2012). O

Monin, B., \& Miller, D. T. (2001). A

Simbrunner, P., \& Schlegelmilch, B. B. (2016), C
Blanken, I., van de Ven, N.. Zeelenberg, M. \& Meijers, M. H. (2014). B

Effron, D. A. Miller, D. T. \& Monin, B. (2012) A

Mazar, N., \& Zhong, C. B. (2010). A

Zhong, C. B., Ku, G., Lount, R. B., \& Murnighan, J. K. (2010). A

Blanken, L., Van de Ven, N., \& Zeelenberg, M.
Clot, S., Grolleau, G., \& Ibanez, L. (2013). B

Clot, S., Grolleau, G., \& I banez, L.

Clot, S., Grolleau, G., \& lbanez, L. (2014). C

Effron, Cameron \& Monin (2009)

Simbrunner, P., \& Schlegelmilch, B. B. (2016), D

Comelissen, G., Karelaia, N., \& Soyer, E. (2013) B

Susemind, M.. \& Hoelz, E. (2014). A

Khan, U., \& Dhar, R. (2006). D
Cain, D. M., Loewenstein, G., \& Moore, D. A. (2011). C

Choi, B., Crandall, C. S., \& La, S. (2014). A

Effron, Cameron \& Monin (2009) A

Effron, D. A. (2014). A

Susewind, M., \& Hoelzl, E. (2014). B

Khan, U,, \& Dhar, R. (2006).

. E Skorinko, J., Hebl, M. R. \& McKenna, C. (2010). D

Effron, D. A. (2014). B

Blanken, L., Van de Ven, N., \& Zeelenberg, M. (2012). K

Blanken, I., Van de Ven,

Comelissen, G., Bashshur, M. R., Rode, J., \& Le Menestrel, M. (2013). C

Blanken, L., Van de Ven, N. \& Zeelenberg, M. (2012). G

(2)

Mann, N. H. \& Kawakami, K (2012). B

Khan, U., \& Dhar, R. (2006). C
Khan, U., \& Dhar, R. (2006).F

Comelissen, G., Karelaia, N., \& Soyer, E. (2013) A

Sachdeva, S., lliev, R., \& Medin, D. L. (2009). C

Simbrunner, P., \& Schlegelmilch, B. B. (2016), E

Cain, D. M. Loewenstein G. \& Moore, D. A. (2005). A

Conway, P., \& Peetz, J. (2012). B

Comelissen, G., Bashshur, M. R., Rode, J., \& Le Menestrel, M. (2013). A

Khan, U., \& Dhar, R. (2006). B

Meijers, M. H. C., Noordewier, M. K., Verlegh, P. W. J., \& Smit, E. G. (2014). C

Blanken, I Van de Ven N \& Z Zeelenberg. M (2012) J

Khan, U., \& Dhar, R. (2006). A
Blanken, I., Van de Ven, N., \& Zeelenberg, M. (2012). L

Kouchaki, M. (2011). D

Bradley-Geist, J. C., King, E. B., Skorinko, J., Hebl, M. R. \& McKenna, C. (2010). B

Simbrunner, P., \& Schlegelmilch, B. B. (2016), A rayban

(2001). B

Conway, P. \& Peetz J. (2012) D

Meijers, M. H. C., Noordewier, M. K., Verlegh, P. W. J., \& Smit, E. G. (2014). B

Comelissen, G., Bashshur, M. R., Rode, J., \& Le Menestrel, M. (2013). B

Blanken, I., Van de Ven, N., \& Zeelenberg, M. (2012). N

Mann, N. H., \& Kawakami, K. (2012).

Jordan J Mullen, E \& Murnighan J K (2011).

Jordan, J., Mullen, E., \& Mumighan, J. K. (2011).

Sachdeva, S., lliev, R., \& Medin, D. L. (2009). B

Jordan, J., Mullen, E., \& Mumighan, J. K. (2011). A

Mazar, N., \& Zhong, C. B. (2010). B

Mazar, N., \& Zhong, C. B. (2010). C

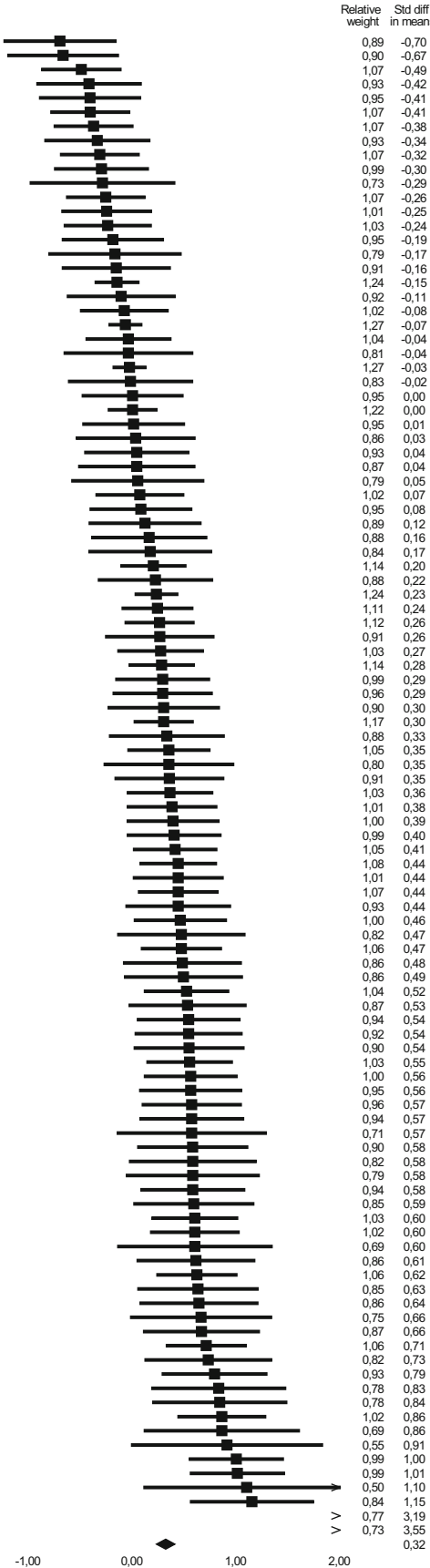

Fig. 1 Forest plot showing the distribution of single effect sizes ranked from smallest to largest. Note for each study the black dot represents its Cohens's d, the lines represent the according confidence interval. Due to space restrictions, two studies are not displayed since they lie outside the scaled interval indicated. Their position is indicated by arrows 
size's $p$-value to a non-significant level (Borenstein et al. 2013). The fail-safe-N for our analysis is 4531 . The test of heterogeneity was significant $(Q=453.377, d f(Q)=105$, $p=0.000$ ), indicating substantial variation between single study outcomes. The $I^{2}$ value indicates that $76.84 \%$ of the variability of single effect size measures is due to systematic between-study differences. We address the identification of sources of variation by both conducting subgroup analyses and meta-regression models employing the moderators described above.

\subsection{Subgroup analyses}

In the subgroup analyses, we analyze each dummy-coded moderator separately. Table 2 depicts detailed results of the analyses. Our findings demonstrate that the licensing effect differs between all three groups of cultural background $(Q(2)=61.404$, $p<0.05)$. North American studies show the highest generalized effect size $(d=$ $0.511, S E=0.074)$, followed by Western European studies $(d=0.236, S E=0.050)$. In South-East Asian studies, the licensing effect in general points into the opposite direction $(d=-0.370, S E=0.087)$. Studies with distinct publication status display significantly different effect sizes $(Q(1)=22.322, p<0.05)$. Published studies $(d=0.492, S E=0.064)$ show bigger licensing effects than unpublished ones $(d=0.089, S E=0.056)$. The type of comparison also influences the size of moral licensing effects significantly $(Q=7.277, p<0.05)$. When compared to an immoral condition $(d=0.641, S E=0.149)$ our analysis demonstrates that moral licensing effects tend to be larger than when compared to a neutral control condition $(d=0.225$, $S E=0.040$ ). The type of decision, i.e. self-related decisions versus society-related ones, does not influence the size of moral licensing effects when looking at the total sample of available studies. In addition to the analysis based on the complete dataset, we ran

Table 2 Subgroup analyses

\begin{tabular}{|c|c|c|c|c|}
\hline & $N$ & Cohen's d, 95\% (CI) & SE & Q-test of heterogeneity \\
\hline Cultural background & & & & $Q=61.404 *, d f(\mathrm{Q})=2$ \\
\hline South-East Asia & 5 & $d=-0.370(-0.54 ;-0.20)$ & 0.087 & \\
\hline Western Europe & 44 & $d=0.236(0.137 ; 0.334)$ & 0.050 & \\
\hline North America & 52 & $d=0.511(0.366 ; 0.657)$ & 0.074 & \\
\hline Type of decision & & & & $Q=1.584, d f(\mathrm{Q})=1$ \\
\hline Self-related & 54 & $d=0.381(0.228 ; 0.535)$ & 0.078 & \\
\hline Society-related & 52 & $d=0.265(0.169 ; 0.361)$ & 0.049 & \\
\hline Comparison & & & & $Q=7.277 *, d f(\mathrm{Q})=1$ \\
\hline Immoral & 26 & $d=0.641(0.349 ; 0.934)$ & 0.149 & \\
\hline Control & 80 & $d=0.225(0.147 ; 0.302)$ & 0.040 & \\
\hline Publication status & & & & $Q=22.322 *, d f(\mathrm{Q})=1$ \\
\hline Unpublished & 45 & $d=0.089(-0.021 ; 0.199)$ & 0.056 & \\
\hline Published & 61 & $d=0.492(0.366 ; 0.618)$ & 0.064 & \\
\hline
\end{tabular}

* Significant at $\alpha=0.05$ 
a subgroup analysis for this specific variable using data from Western countries only. For this additional analysis, we excluded data from South-East Asia for the following reasons: First, how an individual perceives decisions related to one's self or to society is linked to the value this individual ascribes to collectivistic and/or individualistic thinking. Collectivism and individualism are well-known cultural values (Hofstede et al. 2010). Therefore, the cultural background of the samples included might affect the potential influence of the variable type of decision. Second, since we could identify comparatively fewer studies conducted in South-East Asia than studies from Western countries, we were not able to investigate a potential interaction effect between both variables in a statistically meaningful way. Subgroup analysis for the type of decision based on Western samples only showed statistically significant results $(Q=3.911$, $p<0.05)$. Moral licensing effect is higher in self-related $(d=0.480, S E=0.089)$ than in society-related decisions $(d=0.280, S E=0.048)$

Since this factor does not influence the size of moral licensing effects in the total sample of available studies, we exclude it from the further meta-regression analyses.

\subsection{Meta-regression}

We conducted four meta-regression models with the remaining three dummy-coded moderators included in all possible combinations. Table 3 depicts all moderators' $\beta$ values, model test statistics and the overall explained variance metric $R^{2}$ for each model. The model test metrics investigate the Null-hypothesis that simultaneously all coefficients except the intercept are zero. The goodness of fit metrics investigates the Null-hypothesis that the unexplained variance of the model is zero, where $\tau^{2}$ is the variance not explained by the model. The total (i.e. explained and unexplained) between-study-variance is $\tau^{2}=0.1596$, and naturally is the same within all models. All model tests are based on a total sample size of $N=101$. For the meta-regression analyses, we excluded five studies for which we were not able to identify the necessary information in order to allocate the data to a particular category of a moderator. The cultural background moderator consists of three categories (North America, Western Europe and South-East Asia). To obtain $\beta$-values for this moderator, in the metaregression models this factor was split in two parts, each contrasting two groups. The complete moderator's influence (i.e. its influence across all three groups) within models 1, 2 and 3 is $Q_{\text {modell }}(2)=8.31, p=0.016 ; Q_{\text {model } 2}(2)=17.97, p<0.000$; and $Q_{\text {model } 3}(2)=8.26 ; p=0.016$, respectively.

Model 1 consists of cultural background contrasting South-East Asia with Europe $(\beta=0.528 ; S E=0.194 ; z=2.72 ; p=0.006)$ and with North America $(\beta=0.676$; $S E=0.248 ; z=2.72 ; p=0.006)$, type of comparison $(\beta=-0.406 ; S E=0.111$; $z=-3.66 ; p<0.001)$ and publication status $(\beta=0.085 ; S E=0.163 ; z=0.52$; $p=0.603)$. The model explains $22 \%$ of variation between single study outcomes of moral licensing effect sizes $\left(R^{2}=0.22\right)$. Model 2 contains the cultural background contrasting South-East Asia with Europe $(\beta=0.544 ; S E=0.192 ; z=2.84 ; p=$ $0.004)$ and with North America $(\beta=0.757 ; S E=0.191 ; z=3.96 ; p<0.001)$ and type of comparison $(\beta=-0.417 ; S E=0.109 ; z=-3.84 ; p<0.001)$. The model explains $23 \%$ of variation between single studies $\left(R^{2}=0.23\right)$. Model 3 is set up of cultural background contrasting South-East Asia with Europe $(\beta=0.569 ; S E=0.203$; 


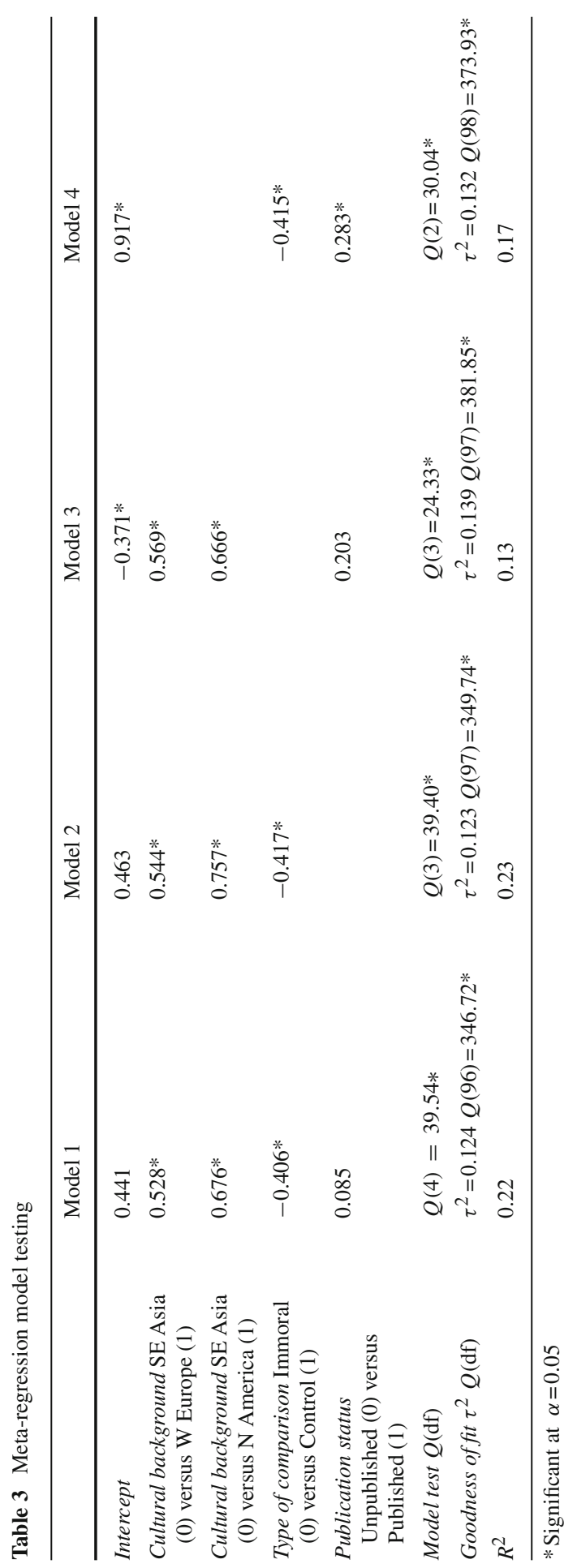


$z=2.81 ; p=0.005)$ and with North America $(\beta=0.666 ; S E=0.259 ; z=2.57$; $p=0.010)$ and publication status $(\beta=0.203 ; S E=0.167 ; z=1.22 ; p=0.223)$. The model explains $13 \%$ of the observed variation between single studies $\left(R^{2}=0.13\right)$. Model 4 incorporates type of comparison $(\beta=-0.415 ; S E=0.113 ; z=-3.68$; $p<0.001)$ and publication status $(\beta=0.283 ; S E=0.092 ; z=3.07 ; p=0.002)$. The model explains $17 \%$ of single study variance $\left(R^{2}=0.17\right)$.

All four models are able to explain a substantial amount of moral licensing effect sizes' variation. However, the model of choice is model 2. First it explains the largest portion of variance and second all coefficients are statistically significant. Interestingly, the publications status is non-significant in two out of three models in which it is contained. When considering publication status with other aspects simultaneously, its ability to explain variation in moral licensing disappears. Both cultural background and type of comparison show a significant and substantial influence on the moral licensing effect in all models in which they are contained.

\section{Discussion}

In the current meta-analysis and meta-regression, we were able to identify two factors-cultural background and type of comparison-which explain a substantial amount of variation in the size of moral licensing effects. Both taken into consideration simultaneously explain $23 \%$ of the total observed variability. Most importantly, we were able to show, for the first time, that cultural background influences the moral licensing effect.

We find a Cohen's $d$ of $0.319,95 \%$ CI $(0.229 ; 0.408)$ as an overall generalized moral licensing effect size that is statistically different from zero. Our fail-safe-N calculation indicates that it is both robust and substantially different from zero. The magnitude of our computed $d$-value is in line with previous findings (Blanken et al. 2015), which identified a statistically non-significantly different, marginally smaller mean effect size of 0.31 .

In the following section, we provide a discussion of the findings regarding the moderators under investigation in our study, highlight both theoretical and managerial implications of our results and present promising future research directions in this area.

\subsection{Cultural background}

Our results show that the effect of moral licensing is stronger in North America compared to Western Europe. Moreover, the moral licensing effect occurs in reversed direction in Southeast Asia. Given the shared historic roots as well as strong cultural, political and economic bonds, North Americans and Western Europeans arguably share a variety of beliefs and fundamental values. However, they differ greatly in some dimensions related to morality, especially in their perceptions about which behavior is judged as being moral or immoral (Pew Research Center 2014). Both regions differ with regard to the meaning they give to the role of religion in their life, with North Americans attaching a substantially greater value on religious beliefs than Western 
Europeans. Moreover, the importance of incorporating unselfishness as a key trait in one's personality structure is valued distinctively higher among North Americans than Western Europeans (World Values Survey 2014). Hence "being a good person" is more important to North American individuals than to Western Europeans. We think that this difference in the overall ascribed importance of morale might explain the moral licensing effect being stronger in North America. With questions of morality being perceived far more pivotal, North Americans presumably see a fluctuation in one's own behavior with regard to moral dimensions as less acceptable than Western Europeans. Vice versa, Western Europeans are more likely to allow themselves to be immoral. Consequently, the value of a license, which enables an individual to behave immoral, is smaller compared to the value a North American individual attributes to this license.

Moral licensing effect is reversed in direction for South-East Asians, indicating that they do not seem to experience moral licensing in the observed studies. Instead, displaying moral behavior increases the likelihood of acting in a moral way subsequently. Hence, for South-East Asians it seems to be more important to maintain consistency in their behavior. This is in line with established psychological theories such as selfperception theory (Bem 1972) or cognitive dissonance theory (Festinger 1957), which stress consistency in an individual's behavior as one key element of human behavior. However, this result seems to mismatch the proposed self-regulation framework (Sachdeva et al. 2009), which serves as a theoretical explanation for moral licensing. According to this framework, individuals establish a moral self-image throughout their lifespan. By displaying moral behavior in a moral licensing experimental setting, they reinforce this moral self-image. This in turn allows individuals to display immoral behavior, since by having reinforced one's own moral self-image; it is not put under threat by displaying immoral behavior subsequently in this very moment. Our results from North America and Western Europe match this explanatory pattern. Regarding our results from South-East Asia, we think cultural theories focusing on human time perception might offer one potential explanation to align our results with the selfregulation framework. More specifically, we consider the concept of Time Orientation as a suitable explanatory route. Time Orientation Theory (Bergadaa 1990) suggests three temporal dimensions - past, present and future time orientation. These temporal dimensions influence human decision-making and judgment processes. Previous research demonstrated the importance of time orientation in both consumer behavior and charitable behavior (Simbrunner et al. 2017). Despite the identification of three distinct temporal dimensions, individuals do not show solely one dimension, but rather embrace each dimension with differing levels of emphasis. Hence, individuals differ in the degree to which they adhere to each of these dimensions. Depending on the dominant time orientations, an individual's behavior is strongly influenced either by past experiences, by current moods or by expectations towards the future (Zimbardo and Boyd 1999). And vice versa weakly pronounced dimensions diminish the respective influence of this source of behavioral guidance. For example, a relatively weakly developed present time orientation implies that recent events do not affect current behavior strongly. Despite being an individual trait, time orientation is also a cultural dimension acquired through socialization processes and hence through interaction with a given cultural environment (Simbrunner et al. 2017). Consequently, members 
of different cultural regions show distinctly pronounced temporal dimensions (e.g. Makri and Schlegelmilch 2017). On average South-East Asians tend to show the weakest pronounced present time orientation compared to members of other world regions (Rojas-Méndez et al. 2002). Thus, recent experiences and recent own behavior is of less relevance as a guiding influence on subsequent behavior for South-East Asians as compared to for example North Americans or Western Europeans. In moral licensing experimental settings, the time span between the moral license-inducing task and the second task in which the degree of morality is measured typically is rather short. Since South-East Asians base their actual behavior to a lesser degree on current circumstances than North Americans or Western Europeans do, but are relatively stronger guided by past experiences and/or future expectations, the need for maintaining consistency in one's moral self-image looms larger. Consequently, after having demonstrated moral behavior, they tend to show behavior that is even more moral subsequently, which is reflected in a "negative" moral licensing effect outcome.

\subsection{Type of comparison}

Contradicting previous findings (Blanken et al. 2015), we find that the comparison between either a moral licensing group and a neutral control group or a moral licensing group and an immoral control group does substantially influences the size of the moral licensing effect. This result is to be expected taking into account the phenomenon of moral cleansing, which is the exact opposite of moral licensing. Displaying or recalling immoral behavior leads to more moral behavior subsequently if compared to a neutral control condition (e.g. Conway and Peetz 2012; Zhong and Liljenquist 2006). Given the existence of moral cleansing, the moral licensing effect is larger when the moral group is compared to an immoral group than when contrasted with a neutral group. Moreover, considering that both approaches represent two distinct methodological approaches, a resulting difference in effect sizes seems to be a logical consequence. We included this variable mostly because-surprisingly-previous research (Blanken et al. 2015) could not identify a systematic influence on the size of moral licensing effects when considering this specific aspect. Having included a more fine-grained set of primary studies in our analyses explains the additional insight we could gain compared to previous studies on this specific issue.

\subsection{Publication status}

In our subgroup analysis, we find that moral licensing effect sizes differ substantially when the publication status is taken into account. In line with previous findings (Blanken et al. 2015), our results show that published data tends to provide bigger effect sizes than unpublished data. However and interestingly, when we look at this aspect whilst simultaneously taking into consideration the influence of both cultural background and type of comparison, the publication status' influence disappears. Hence, the difference in effect sizes between published and unpublished data is better explained by the cultural environment these studies were conducted in than by whether they are published or not. Probably, whether a data set is published or unpublished is not a 
"characteristic" per se but instead might rather be a reflection of a surprising result that led to the consequence of these studies remaining unpublished. The question remains, whether studies are unpublished because of unexpected results due to sampling errors, or whether the unexpected results in fact are part of reality, which do not get published because they contradict mainstream assumptions.

\subsection{Type of decision}

We investigated the type of decision, i.e. if the decision in the second task is rather of benefit for oneself or for society, as a potential moderator, but could not identify a systematic influence of this aspect on moral licensing effects in the total sample of available studies. However, when looking at data from Western countries only, we identify a systematic variation in the size of moral licensing effects with regard to this issue. Moral behavior influences subsequent decisions that result in a self-benefit or include a self-indulgent component more strongly than decisions that benefit society. Our results support the assumption that experiencing a boost in one's moral selfconcept increases the likelihood of seeking self-gratification.

When looking at this aspect by including data from South-East Asia as well, the systematic variation between these two distinct types of decision disappears. We assume an interactional effect between the type of decision and the cultural background. How an individual perceives decisions related to one's self or to society, respectively, is linked to the value this individual ascribes to collectivistic and/or individualistic thinking. Due to a comparatively small number of samples from non-Western countries, we refrained from further investigating this interactional pattern.

\subsection{Future research directions}

We were able to detect, for the first time, two study-specific factors, which systematically influence the strength of moral licenses. Cultural background and type of comparison taken into consideration simultaneously are able to explain almost a quarter of observed variation in the magnitude of moral licensing effects. However, an even bigger proportion of variability remains unexplained. Therefore, we strongly encourage scholars to work further on closing this gap in existing research. Based on our findings, we consider the following as promising future research avenues.

Our study demonstrates, for the first time, that moral licensing effect is dependent on the cultural background of participants. Having identified this effect between Western European, North American and South-East Asian samples, we encourage future research to investigate cultural differences in further cultural regions. Since this effect is by definition connected to individuals' understandings of morale, it ultimately is related to religion. Independently from the fact whether a person considers herself/himself as a religious person, religion undoubtedly shaped - from a historical perspective-how a society understands morale. Under this premise, we strongly encourage a systematic investigation of the dynamics of moral licensing effect in, for example, Muslim, Hindu or Shinto societies. 
Additionally, we use the cultural dimension of Time Orientation as theoretical framework to explain differences in the dynamics of moral licensing effects between culturally distinct regions. Future research should further investigate this conceptualization in primary studies and potentially investigate alternative concepts of time, such as polychromic/monochromic time perspectives etc.

Furthermore, we identified cultural differences between Europe and North America, thereby highlighting the importance of cross-cultural research between seemingly closely related cultural regions. Hence, we encourage future research in the area of behavioral economics to consider specifically potential differences between these two rather similar cultures.

We suggest a potential interaction between the cultural background on the one hand and the type of decision, i.e. self-related or society-related, on the other hand. Based on Hofstede et al. (2010) collectivism/individualism dichotomy, we suggest for instance that society-related decisions yield different outcomes in rather collectivistic societies as compared to individualistic societies.

Previous research by Blanken et al. (2015) investigated several potential moderators as outlined above but could not identify any substantial influences. Prior research relied on data from Western countries only. In the light of cross-cultural differences, we strongly encourage future research to re-examine existing attempts to identify moderating aspects on moral licensing in a cross-cultural setting.

Both our study and previous contributions of Blanken et al. (2015) explain variation in moral licensing effects. However, we realize that all of the investigated potential moderators are external factors, such as variations of the stimulus material used in primary studies and/or differences in methodological approaches. We assume that less "obvious" aspects might offer suitable explanations for the effect's variability, such as personality traits of participants. As suggested by our results regarding the cultural background, general attitudes towards morality or individual time orientation could offer further insights into underlying mechanisms of the effectiveness of moral licensing. Thus, we would like to encourage future research to focus on the potential impact of personality-related aspects and individual traits on moral licensing. The lack of a systematic investigation of personality facets is a striking gap in existing literature.

Furthermore, we notice that most findings from studies on moral licensing lack a direct applicability for managers and/or marketers. For example, most experimental designs rely on fictitious brands; employ forced choice designs or use two tasks, which in terms of content are rather unrelated. We understand that experimental settings are often designed in a way that maximizes the likelihood of the occurrence of the effect under investigation. This approach is of course highly desirable at an early stage of research on a given effect, since the focal goal of studies often is to identify the existence of the effect per se. However, we think that moral licensing today is a wellestablished effect and research in this area has reached a stage at which it is highly recommended to identify circumstances under which this effect can be applied in practice. We therefore explicitly call for future research to investigate moral licensing under settings, which are (more or less) easily transferrable into real-life marketing and/or management measures.

Given the nature of meta-analyses, we were only able to use information provided in primary data, both published and unpublished. Despite having exercised great care on 
data search and appraisal, we are aware that the existence of additional, non-identified data is probable. Therefore, we encourage a repetition of our analyses with additional data, especially from countries not included in this analysis.

\subsection{Conclusion}

We are able to identify aspects, which explain a substantial amount of variation in the size of moral licensing effects. From a theoretical perspective, our research contributes to the extant knowledge on the dynamics in human decision-making processes by identifying key moderators of moral licensing. Most importantly, we demonstrate the existence of cross-cultural differences between three distinct world regions. From a managerial perspective, marketers wishing to capitalize on the moral licensing phenomenon should carefully monitor and (if possible) plan the sequence, in which product offerings are being presented to customers. Our findings demonstrate that due to cross-cultural differences, marketing measures building on moral licensing will lead to different revenues in distinct markets. Moral licensing suggests, for example, that purchasing a green product (a positive moral act) increases the likelihood of purchasing a luxury product (a self-indulgent act) subsequently. Based on our findings, this effect is bigger in North America than in Europe. Additionally, our findings suggest that in South-East Asia, purchasing a green product increases the likelihood of purchasing another green product and vice versa decreases the likelihood of choosing a self-indulgent option.

Acknowledgements Open access funding provided by Vienna University of Economics and Business (WU).

Open Access This article is distributed under the terms of the Creative Commons Attribution 4.0 International License (http://creativecommons.org/licenses/by/4.0/), which permits unrestricted use, distribution, and reproduction in any medium, provided you give appropriate credit to the original author(s) and the source, provide a link to the Creative Commons license, and indicate if changes were made.

\section{References}

Batson CD, Thompson ER (2001) Why don't moral people act morally? Motivational considerations. Curr Dir Psychol Sci 10:54-57

Baumann DJ, Cialdini RB, Kenrick DT (1981) Altruism as hedonism: helping and self-gratification as equivalent responses. J Personal Soc Psychol 40(6):1039-1046

Bem DJ (1972) Self-perception theory. Adv Exp Soc Psychol 6:1-62

Bergadaa MM (1990) The role of time in the action of the consumer. J Consum Res 17(3):289-302

Bond R, Smith PB (1996) Culture and conformity: a meta-analysis of studies using Asch's (1952b, 1956) line judgment task. Psychol Bull 119:111-137

Borenstein M, Hedges LV, Higgins JPT, Rothstein HR (2013) Introduction to meta-analysis. Wiley, Chichester

**Blanken I, Van de Ven N, Zeelenberg M (2012) A variety of studies on the self-licensing effect. Unpublished raw data

*Blanken I, van de Ven N, Zeelenberg M, Meijers MH (2014) Three attempts to replicate the moral licensing effect. Soc Psychol 45(3):232-238

Blanken I, van de Ven N, Zeelenberg M (2015) A meta-analytic review of moral licensing. Personal Soc Psychol Bull 41(4):540-558 
*Bradley-Geist JC, King EB, Skorinko J, Hebl MR, McKenna C (2010) Moral credentialing by association: the importance of choice and relationship closeness. Personal Soc Psychol Bull 36(11):1564-1575

*Brown RP, Tamborski M, Wang X, Barnes CD, Mumford MD, Connelly S, Devenport LD (2011) Moral credentialing and the rationalization of misconduct. Ethics Behav 21(1):1-12

*Cain DM, Loewenstein G, Moore DA (2005) The dirt on coming clean: perverse effects of disclosing conflicts of interest. J Legal Stud 34(1):1-25

*Cain DM, Loewenstein G, Moore DA (2011) When sunlight fails to disinfect: understanding the perverse effects of disclosing conflicts of interest. J Consum Res 37(5):836-857

Cascio J, Plant EA (2015) Prospective moral licensing. Does anticipating doing good later allow you to be bad now? J Exp Soc Psychol 56:110-116

*Choi B, Crandall CS, La S (2014) Permission to be prejudiced: legitimacy credits in the evaluation of advertisements. J Appl Soc Psychol 44(3):190-200

*Clot S, Grolleau G, Ibanez L (2013a) Self-licensing and financial rewards: is morality for sale? Econ Bull 33:2298-2306

**Clot S, Grolleau G, Ibanez L (2013b) Anti-social behavior and self-licensing: Experimental evidence from a gangster game. In: Presented at 30. Journées de Micraoeconomie Appliquée, Nice, FRA

*Clot S, Grolleau G, Ibanez L (2014) Smug alert! Exploring self-licensing behavior in a cheating game. Econ Lett 123:191-194

Colby A, Damon W (1993) The uniting of self and morality in the development of extraordinary moral commitment. The Moral Self, 149-174

Comprehensive Meta-Analysis (Version 3) Computer software. (2014). Englewood, NJ: Biostat. https:// www.meta-analysis.com/

*Conway P, Peetz J (2012) When does feeling moral actually make you a better person? Conceptual abstraction moderates whether past moral deeds motivate consistency or compensatory behavior. Personal Soc Psychol Bull 38:907-919

Cooper H (2010) Research synthesis and meta-analysis. A step-by-step approach. Sage, Thousand Oaks

*Cornelissen G, Bashshur MR, Rode J, Le Menestrel M (2013a) Rules or consequences? The role of ethical mind-sets in moral dynamics. Psychol Sci 24(4):482-488

**Cornelissen G, Karelaia N, Soyer E (2013b) Clicktivism or slacktivism? The ironic effects of symbolic prosocial behavior. In: Paper presented at society for the advancement of judgment and decision making studies, Granada, Spain

Dahl DW, Honea H, Manchanda RV (2003) The nature of self-reported guilt in consumption contexts. Mark Lett 14(3):159-171

Dana J, Weber RA, Kuang JX (2007) Exploiting moral wiggle room: experiments demonstrating an illusory preference for fairness. Econ Theory 33:67-80

*Effron DA (2014) Making mountains of morality from molehills of virtue: threat causes people to overestimate their moral credentials. Personal Soc Psychol Bull 40(8):972-985

*Effron DA, Cameron JS, Monin B (2009) Endorsing Obama licenses favoring whites. J Exp Soc Psychol 45:590-593

*Effron DA, Monin B, Miller DT (2012) Inventing racist roads not taken: the licensing effect of immoral counterfactual behaviors. J Personal Soc Psychol, 103: 916-932

Festinger L (1957) A theory of cognitive dissonance. Stanford University Press, Stanford

Heider F (1946) Attitudes and cognitive organization. J Psychol 21:107-112

Hofstede G, Hofstede GJ, Minkov M (2010) Cultures and organizations: software of the mind. McGraw-Hill, New York

*Jordan J, Mullen E, Murnighan JK (2011) Striving for the moral self: the effects of recalling past moral actions on future moral behavior. Personal Soc Psychol Bull 37:701-713

Khaleque A, Rohner RP (2002) Reliability of measures assessing the pancultural association between perceived parental acceptance-rejection and psychological adjustment: A meta-analysis of cross-cultural and intracultural studies. J Cross Cult Psychol 33:87-99

*Khan U, Dhar R (2006) Licensing effect in consumer choice. J Mark Res 43(2):259-266

Kivetz R, Simonson I (2002) Earning the right to indulge: effort as a determinant of customer preferences toward frequency program rewards. J Mark Res 39(2):155-170

*Kouchaki M (2011) Vicarious moral licensing: the influence of others' past moral actions on moral behavior. J Pers Soc Psychol 101(4):702

**Leonard B (2012) Unpublished raw data

Lipsey MW, Wilson DB (2001) Practical meta-analysis. Sage, Thousand Oaks, CA 
Makri K, Schlegelmilch BB (2017) Time orientation and engagement with social networking sites: a crosscultural study in Austria, China and Uruguay. J Bus Res, in press. doi:10.1016/j.jbusres.2017.05. 016

*Mann NH, Kawakami K (2012) The long, steep path to equality: progressing on egalitarian goals. J Exp Psychol Gen 141(1):187

*Mazar N, Zhong CB (2010) Do green products make us better people? Psychol Sci 21:494-498

**Meijers MHC, Noordewier MK, Verlegh PWJ, Smit EG (2014) Identity relevance moderates the licensing effect (Chapter from doctoral dissertation)

Merritt AC, Effron DA, Fein S, Savitsky KK, Tuller DM, Monin B (2012) The strategic pursuit of moral credentials. J Exp Soc Psychol 48:774-777

Mezulis AH, Abramson LY, Hyde JS, Hankin BL (2004) Is there are universal positivity bias in attributions? A meta-analytic review of individual, developmental and cultural differences in the self-serving attribution bias. Psychol Bull 130:711-747

*Monin B, Miller DT (2001) Moral credentials and the expression of prejudice. J Pers Soc Psychol 81(1):33

Pew Research Center, Washington D.C. (2014) Global views on morality. http://www.pewglobal.org/2014/ 04/15/global-morality/ retrieved 11.12.2016

Rojas-Méndez JI, Davies G, Omer O, Chetthamrongchai P, Madran C (2002) A time attitude scale for cross cultural research. J Glob Mark 15(3-4):117-147

Rosenthal R (1979) The 'file drawer problem' and the tolerance for null results. Psychol Bull 86(3):638-641

*Sachdeva S, Iliev R, Medin DL (2009) Sinning saints and saintly sinners the paradox of moral selfregulation. Psychol Sci 20(4):523-528

Samson A (Ed) (2016) The behavioral economics guide 2016 (with an introduction by Gerd Gigerenzer). Retrieved from http://www.behavioraleconomics.com

**Schüler S, Lehnhardt N, Huber M (2012) Can priming prosocial values eliminate the moral licensing effect? Unpublished manuscript, Technische Universität Dresden, Germany

*Simbrunner P, Schlegelmilch BB (2016) A variety of studies on the moral licensing effect. Unpublished raw data

Simbrunner P, Makri K, Schlegelmilch BB (2017) Time orientation: the role of culture and its marketing applications. Transf Werbeforsch Prax 63(1):17-21

**Spektor M (2014) Unpublished raw data

*Susewind M, Hoelzl E (2014) A matter of perspective: why past moral behavior can sometimes encourage and other times discourage future moral striving. J Appl Soc Psychol 44(3):201-209

Szőcs I, Schlegelmilch BB, Rusch T, Shamma HM (2016) Linking cause assessment, corporate philanthropy, and corporate reputation. J Acad Mark Sci 44(3):376-396

Thaler RH (1985) Mental accounting and consumer choice. Mark Sci 4:199-214

**Thomas, JS, Showers, C (2012). Unpublished raw data

Twenge JM, Campbell WK (2002) Self-esteem and socioeconomic status: a meta-analytic review. Personal Soc Psychol Rev 6:59-71

Varadarajan PR, Menon A (1988) Cause-related marketing: a coalignment of marketing strategy and corporate philanthropy. J Mark 52(3):58-74

Watkins D (2001) Correlates of approaches to learning: a cross-cultural meta-analysis. In: Sternberg R, Zhang L (eds) Perspectives on thinking, learning and cognitive styles. Erlbaum, Mahwah, pp 165-195

Ward M (2014) Cognition, culture, and charity: Sociolinguistics and "donor dissonance" in a Baptist denomination. Voluntas 26(2):574-603

World Values Survey - Wave 6 2010-2014 (2014) Official aggregate v.20150418. World values survey association. Aggregate File Producer: Asep/JDS, Madrid SPAIN. www.worldvaluessurvey.org retrieved 11.12.2016

*Young L, Chakroff A, Tom J (2012) Doing good leads to more good: the reinforcing power of a moral self-concept. Rev Philos Psychol 3(3):325-334

*Zhong CB, Liljenquist KA (2006) Washing away your sins: threatened morality and physical cleansing. Science 313:1451-1452

Zhong CB, Ku G, Lount RB, Murnighan JK (2010) Compensatory ethics. J Bus Ethics 92(3):323-339

Zimbardo PG, Boyd JN (1999) Putting time in perspective: a valid, reliable individual-differences metric. J Pers Soc Psychol 77(6):1271-1288

References marked with an asterisk are included in the Meta-Analysis. Those marked with two asterisks are unpublished data, which were reported in Blanken et al. (2015) and were included in our analyses as well 